\title{
COMUNICACIÓN
}

\section{Infecciones por protozoos y helmintos intestinales en pre-escolares y escolares de la Comuna de Colina, Santiago, Chile. 2003}

\author{
RUBEN MERCADO*, DOUGLAS CASTILLO*, VÍCTOR MUÑOZ*, \\ LEA SANDOVAL*, MARÍA ISABEL JERCIC**, LUIS CARLOS GIL***, \\ MARLENE T. UETA**** y HUGO SCHENONE*.
}

\section{INTESTINAL PROTOZOA AND HELMINTH INFECTIONS IN PRE-SCHOOL AND ELEMENTARY SCHOOL-CHILDREN FROM COLINA COUNTY, SANTIAGO, CHILE, 2003}

In may 2003 an epidemiologic-parasitological survey was carried out in 206 pre-school and schoolchildren from San Vicente de Lo Arcaya School in Colina, a semi-rural county of the Santiago Province in the Metropolitan Region of Chile.The most frequent parasitic elements were: Blastocystis hominis - in men and women respectively - 38.8\% and 44.4\%, Giardia intestinalis with $9.5 \%$ and $16.2 \%$ and Enterobius vermicularis with $12.9 \%$ and $10.0 \%$. It is notewortly that the infected children were asymptomatic Cryptosporidium parvum was not observed and an ELISA test for Strongyloides stercoralis infections was positive in one children.This study confirms that intestinal parasitic infections in children remain with a high prevalence in Chile.

Key words: Survey, Human enteroparasites, Protozoan, Helminth, Chile.

\section{INTRODUCCIÓN}

Colina es una comuna semirural localizada en el extremo noreste de la Región Metropolitana de Chile. El área geográfica abarca $955 \mathrm{~km}^{2}$ e incluye principalmente terrenos agrícolas. La población escolar pre-básica y básica que asiste a establecimientos educacionales municipalizados de Colina al año 2003 es de 971 y 5.533 escolares respectivamente, con un total de 6.504. La mayor parte de la población (70\%) reside en una zona urbana central, densamente poblada. En Colina existen 13 escuelas de enseñanza pre-básica y básica. Entre éstas se encuentra la Escuela San Vicente de Lo Arcaya que está ubicada en el extremo sur de la comuna y su matrícula la constituyen 292 niños.

Según la Organización Mundial de la Salud, las poblaciones infantiles en edad escolar son más vulnerables a los agentes infecciosos, entre ellos

* Unidad de Parasitología Básico-Clínica, Instituto de Ciencias Biomédicas (ICBM), Facultad de Medicina, Universidad de Chile.

** Laboratorio de Referencia de Parasitología, Instituto de Salud Pública de Chile.

*** Servicio de Gastroenterología, Hospital Clínico José Joaquín Aguirre, Universidad de Chile.

**** Departamento de Parasitología, Instituto de Biología, UNICAMP, Campinas, Sao Paulo, Brasil. Email: rmercado@machi.med.uchile.cl 
los parasitarios, y su adecuado desarrollo está condicionado por la contaminación del medio ambiente y la seguridad de los alimentos que consumen ${ }^{1}$.

Debido a que las enteroparasitosis son frecuentes, especialmente en la población infantil y a que están estrechamente ligadas a las condiciones de vida de las comunidades, especialmente de bajo nivel socio-económico, inadecuado saneamiento básico ambiental y condiciones geoclimáticas (suelos y humedad), se realizó el presente estudio que abarcó a la mayoría de la población escolar del referido establecimiento. Por otra parte, en Chile hay carencia de información epidemiológica actualizada acerca de las enteroparasitosis, ya que son escasas las publicaciones al respecto ${ }^{2-4}$.

La finalidad de este estudio es aportar nuevos antecedentes acerca del parasitismo intestinal de los niños de una comuna semirural de Chile, que permita actualizar las estadísticas epidemiológicas en este grupo etáreo de la población.

\section{MATERIAL Y MÉTODO}

En mayo del 2003 se practicaron exámenes parasitológicos a 206 niños (116 hombres, 90 mujeres) que asistían a la Escuela San Vicente de Lo Arcaya de la Comuna de Colina. Las edades oscilaban entre 4 y 14 años.

Todos los niños que participaron en el estudio eran aparentemente sanos y habían concurrido habitualmente a la escuela durante la semana anterior a la realización del estudio.

Los padres de los niños asistieron a una charla informativa, después de la cual dieron su consentimiento por escrito para que sus hijos participaran en el estudio.

De cada niño se obtuvo una muestra de deposiciones la que se depositó en frascos plásticos que contenían $15 \mathrm{ml}$ de formol-sal. Además, se tomó de la región perianal una muestra con una cinta adhesiva trasparente para el diagnóstico de infecciones por Enterobius vermicularis ${ }^{5}$. También se tomó a cada niño una muestra de sangre por punción digital, la que se depositó en papel filtro para la búsqueda de anticuerpos anti Strongyloides stercoralis.

En el laboratorio cada muestra de deposiciones se procesó por el método de Telemann modificado para pesquisar quistes de protozoos y huevos de helmintos ${ }^{6}$. Para la detección de
Cryptosporidium parvum se prepararon extendidos de cada sedimento de las muestras de heces en portaobjetos, los que fueron teñidos con aureamina y observados en un microscopio de luz $\mathrm{UV}^{7}$. La búsqueda de anticuerpos anti $S$. stercoralis se efectuó mediante ELISA Ig G de acuerdo a lo descrito previamente ${ }^{8}$.

Se calculó la significación estadística de las diferencias de frecuencias de infección mediante el estadígrafo Chi cuadrado de EPIINFO 6.0.

\section{RESULTADOS}

Al momento de efectuar la encuesta se pudo observar que 115 de los 206 niños presentaron parásitos y/o comensales intestinales en los exámenes practicados, un 55,8\% del total.

En la Tabla 1 se presentan los resultados observados en los niños agrupándolos en dos grupos etáreos: pre-escolares $(4<6$ años $)$ y escolares (> 6 -14 años). Con relación a los parásitos intestinales la mayor prevalencia correspondió a las infecciones por el protozoo Blastocystis hominis, con un 41,3\%. De los protozoos comensales Entamoeba coli fue la espécie más frecuentemente hallada con un $17,0 \%$. Las frecuencias de infección observadas en los escolares para B. hominis, Giardia intestinalis, Entamoeba histolytica/dispar y E. vermicularis fueron mayores que las encontradas en los pre-escolares, destacando las producidas por B. hominis cuyas diferencias fueron significativas $(\mathrm{p}<0,01)$.

En la Tabla 2 se muestran las frecuencias de infección por protozoos y helmintos, de acuerdo al sexo de los niños. Las diferencias observadas en los porcentajes de infección según las especies encontradas no fueron estadísticamente significativas.

C. parvum no fue encontrado en los niños estudiados. Mediante ELISA Ig G se detectó un caso positivo para S. stercoralis.

\section{DISCUSIÓN}

Los resultados obtenidos muestran que las enteroparasitosis continúan siendo de elevada frecuencia en la población pre-escolar y escolar de Chile. Destaca como el parásito más frecuentemente hallado el $B$. hominis con una prevalencia global de $41,3 \%$ y de $47,3 \%$ en los escolares. Este índice se mantiene en el tiempo y 
Protozoos y helmintos intestinales en niños de Colina, Santiago, Chile - R. Mercado et al.

Tabla 1. Frecuencia de infección por protozoos y helmintos intestinales en pre-escolares y escolares de Colina, según grupos de edades

\begin{tabular}{|c|c|c|c|c|c|c|}
\hline \multirow[t]{3}{*}{ Especies } & \multicolumn{4}{|c|}{ Grupos de edades (años) } & \multirow{2}{*}{\multicolumn{2}{|c|}{$\begin{array}{l}\text { Total } \\
(206)\end{array}$}} \\
\hline & \multicolumn{2}{|c|}{$4<6(41)$} & \multicolumn{2}{|c|}{$>6-15(165)$} & & \\
\hline & $\mathbf{n}$ & $\%$ & $\mathbf{n}$ & $\%$ & $\mathbf{n}$ & $\%$ \\
\hline Blastocystis hominis & 7 & 17,1 & 78 & $47,3 *$ & 85 & 41,3 \\
\hline Entamoeba coli & 7 & 17,1 & 28 & 17,0 & 35 & 17,0 \\
\hline Giardia intestinalis & 4 & 9,8 & 22 & 13,3 & 26 & 12,6 \\
\hline Endolimax nana & 4 & 9,8 & 15 & 9,1 & 19 & 9,2 \\
\hline Entamoeba histolytica/dispar & 1 & 2,4 & 7 & 4,2 & 8 & 3,9 \\
\hline Iodamoeba buetschlii & 0 & 0,0 & 2 & 1,2 & 2 & 1,0 \\
\hline Chilomastix mesnili & 0 & 0,0 & 1 & 0,6 & 1 & 0,5 \\
\hline Enterobius vermicularis & 3 & 7,3 & 21 & 12,7 & 24 & 11,7 \\
\hline
\end{tabular}

$* \mathrm{p}<0,01$

Tabla 2. Frecuencia de infección por protozoos y helmintos intestinales en pre-escolares y escolares de la comuna de Colina, según sexo

\begin{tabular}{lrrrr}
\hline Espécies & \multicolumn{2}{c}{$\begin{array}{c}\text { Mujeres } \\
(\mathbf{9 0 )}\end{array}$} & \multicolumn{2}{c}{$\begin{array}{c}\text { Hombres } \\
(\mathbf{1 1 6})\end{array}$} \\
& $\mathbf{n}$ & $\mathbf{\%}$ & $\mathbf{n}$ & $\boldsymbol{\%}$ \\
\hline Blastocystis hominis & 40 & 44,4 & 45 & 38,8 \\
Entamoeba coli & 18 & 20,0 & 17 & 14,7 \\
Giardia intestinalis & 15 & 16,7 & 11 & 9,5 \\
Endolimax nana & 7 & 7,8 & 12 & 10,3 \\
Entamoeba histolytica/dispar & 3 & 3,3 & 5 & 4,3 \\
Iodamoeba buetschlii & 1 & 1,1 & 1 & 0,9 \\
Chilomastix mesnili & 0 & 0,0 & 1 & 0,9 \\
Enterobius vermicularis & 9 & 10,0 & 15 & 12,9 \\
\hline
\end{tabular}

es similar al encontrado en 1989 para escolares de Santiago $(51,8 \%)^{2}$, al observado en 1993 en escolares rurales de la isla grande de Chiloé $(62,3 \%)^{3}$ y al encontrado en 1997 en escolares de sectores ribereños en Valdivia $(50,1 \%)^{4}$. Con relación a dicho protozoo se ha podido recientemente describir una forma quística similar a la de otros protozoos intestinales, lo que explicaría que su transmisión se produzca por ingestión de aguas contaminadas con deposiciones humanas o de frutas y verduras que crecen a ras del suelo y que fueron regadas con dichas aguas. Por otra parte, la patogenicidad de $B$. hominis se mantiene sin definir ${ }^{9}$. Este estudio fue realizado en niños aparentemente sanos que al momento de la investigación no presentaban manifestaciones digestivas.

La presencia de otros parásitos y comensales intestinales en los exámenes procesados como
E. coli y E. nana muestran claramente que la población infantil de la región central de Chile esta constantemente expuesta a adquirir infecciones intestinales mediante mecanismos que impliquen contaminación con heces humanas de los alimentos o aguas de bebida.

En niños pre-escolares y escolares de Colina, E. vermicularis se observó con una frecuencia global de infección de 11,7\%. En escolares de Santiago aparentemente sanos se encontró este nematodo en el 39,9\% en $1989^{2}$ y en escolares de la isla grande de Chiloé en el 20,1\% ${ }^{3}$. Por otra parte, en escolares del sector norte de la ciudad de Santiago que concurrieron a consultorios ambulatorios y les fue solicitado un examen de Graham para pesquisa de E. vermicularis se encontró una frecuencia de $35,2 \%{ }^{10}$. Aunque en el presente estudio se observó comparativamente una menor frecuencia de infección por $E$. 
vermicularis que la reportada en los escolares en publicaciones previas, es posible explicar esta diferencia por que se usó sólo una toma de muestras para detectar el parásito, por razones de logística del proceso de muestreo. En los otros estudios mencionados para detectar huevos de E. vermicularis se han usado tres o cinco cintas adhesivas tomadas en tres o cinco días consecutivos, lo que aumenta ampliamente la probabilidad de diagnosticar la parasitosis ${ }^{11}$.

C. parvum no fue encontrado en niños preescolares y escolares lo que corrobora anteriores estudios realizados para detectar este protozoo en diversas ciudades del país. En Chile, en individuos inmunocompetentes las infecciones por C. parvum afectan mayoritariamente a los lactantes y son escasas o nulas en personas de edades mayores ${ }^{12}$.

Recientemente se introdujo en Chile la reacción de ELISA para el diagnóstico serológico de infecciones por $S$. stercoralis. Usando esta metodología se pudo detectar que uno de los escolares presentaba una reacción positiva. El estudio etiológico de esta infección se debe complementar con la búsqueda de larvas del parásito en exámenes de deposiciones, así como la presencia de eosinofilia sanguínea. ELISA para S. stercoralis es una prueba altamente especifica y sensible que contribuirá significativamente al conocimiento de la epidemiología de esta enteroparasitosis ${ }^{8}$.

De acuerdo a los resultados obtenidos en el presente estudio, las infecciones intestinales por parásitos intestinales de los pre-escolares y escolares continúan siendo de elevada frecuencia en Chile.

\section{RESUMEN}

En mayo de 2003 se efectuó una encuesta epidemio-parasitológica en pre-escolares y escolares de la Escuela San Vicente de Lo Arcaya de Colina, comuna semirural de la Provincia de Santiago ubicada en la Región Metropolitana de Chile. Hay que destacar que los niños estudiados eran asintomáticos.

Los elementos parasitarios más frecuentemente encontrados fueron: Blastocystis hominis - en hombre y mujeres respectivamente - $38,8 \%$ y $44,4 \%$, Giardia intestinalis con $9,5 \%$ y $16,2 \%$ y
Enterobius vermicularis con $12,9 \%$ y $10,0 \%$.

Cryptosporidium parvum no fue encontrado y ELISA para Strongyloides stercoralis resultó positiva en un niño.

\section{REFERENCIAS}

1.- WORLD HEALTH ORGANIZATION. World Health Assembly. Resolution WHA 54. 19. 2001.

2.- MERCADO R, ARAVENA A, ARIAS B et al. Frecuencia de infección por enteroparásitos en escolares de Santiago de Chile 1988-1989. Bol Chil Parasitol 1989; 44: 89-91.

3.- MEJIAS G. Intestinal parasite infections in rural students of Chiloé archipelago, X Region, Chile. Bol Chil Parasitol 1993, 48: 28-9.

4.- TORRES P, OTTH P, MONTEFUSCO A et al. Infecciones por protozoos y helmintos intestinales en escolares de sectores ribereños, con distinto nivel de contaminación fecal, del río Valdivia, Chile. Bol Chil Parasitol 1997; 52: 3-11.

5.- GRAHAM DF. A device for the diagnosis of Enterobius vermicularis infection. Am J Trop Hyg 1941; 21: 1501.

6.- DOREN G, GALDAMES M, SILVA R. Algunas consideraciones sobre el rendimiento de las técnicas de diagnóstico de enteroparásitos. Bol Chil Parasitol 1958; 13: 42-4.

7.- BAXBY D, BLUNDELL N, HART C. The development and performance of a simple, sensitive method for the detection of Cryptosporidium oocysts in faeces. J Hyg 1984; 93: 317-23.

8.- MERCADO R, JERCIC MI, TORRES P et al. Immunodiagnosis of Strongyloides stercoralis infections in Chile using an ELISA test. Rev Méd Chile 2002; 130: 1358-64.

9.- TAN KS, SINH M, YAP EH. Recent advances in Blastocystis hominis research: hot spot in terra incognita. Int J Parasitol 2002; 15: 789-804.

10.- MERCADO R, GARCIA M. Various epidemiological aspects of Enterobius vermicularis infections in inpatients served at outpatinets clinics and hospitals from the north section of Santiago, Chile. Bol Chil Parasitol 1996; 51: 91-4.

11.- SCHENONE H, ARIAS B, GALDAMES $M$ et al. Rendimiento de los exámenes seriados en el diagnóstico de laboratorio de la infección por Enterobius vermicularis. Bol Chil Parasitol 1970; 25: 102-17.

12.- MERCADO R. Epidemiological and diagnostic aspects of human criptosporidiasis in Chile. Bol Chil Parasitol 1992; 47: 30-2.

Agradecimientos: Al Sr. Juan Baldeig Z. Director de Educación de la Corporación Municipal de Desarrollo Social de Colina, a la Directora Sra. María Eugenia Andaur A., profesores y administrativas de la Escuela San Vicente de Lo Arcaya por su valiosa colaboración para realizar este estudio. 